https://helda.helsinki.fi

Comparison of intubation and tracheotomy in patients with deep neck infection

\title{
Tapiovaara, Laura
}

2017-10

Tapiovaara , L, Back , L \& Aro , K 2017 , ' Comparison of intubation and tracheotomy in patients with deep neck infection ' , European Archives of Oto-Rhino-Laryngology , vol. 274 , no. 10 , pp. 3767-3772 . https://doi.org/10.1007/s00405-017-4694-5

http://hdl.handle.net/10138/245627

https://doi.org/10.1007/s00405-017-4694-5

publishedVersion

Downloaded from Helda, University of Helsinki institutional repository.

This is an electronic reprint of the original article.

This reprint may differ from the original in pagination and typographic detail.

Please cite the original version. 


\title{
Comparison of intubation and tracheotomy in patients with deep neck infection
}

\author{
Laura Tapiovaara $^{1}\left(\mathbb{D} \cdot\right.$ Leif Bäck $^{1} \cdot$ Katri Aro $^{1,2}$
}

Received: 6 April 2017 / Accepted: 27 July 2017 / Published online: 5 August 2017

(c) Springer-Verlag GmbH Germany 2017

\begin{abstract}
Possible airway compromise further complicates treatment of deep neck infections (DNI). Airway management is crucial, but factors affecting the method of choice are unclear. We retrospectively evaluated adult DNIs in a single tertiary center covering 10 years, with special attention on airway management. Patient data were retrieved from electronic data files from 2007 to 2016, and included adult patients with DNI operated through the neck. Of the 202 patients, 127 (63\%) were male, with a median age of 47 years. Odontogenic $(n=74 ; 35 \%)$ infection was the most common etiology. Intubation was the most common method of airway management $(n=165 ; 82 \%)$, and most patients $(n=102 ; 50 \%)$ were extubated immediately after surgery. Tracheotomy was performed primarily for 35 (17\%) patients, and secondarily for $25(15 \%)$. Two patients were managed in local anesthesia. Altogether $80(40 \%)$ patients required care in the intensive care unit for a median of 7 days. Median hospital stay was 6 days for intubated patients and 10 days for primarily tracheotomized ( $p=0.036$ ). DNI extended to the mediastinal space in $25(12 \%)$ patients, most of whom with odontogenic infection (48\%), and necrotizing fasciitis $(32 \%)$. Odontogenic infection was the most common etiology for DNI with increased risk for mediastinal involvement. Intubation was most common type of airway management with high success in immediate extubation after surgery. The
\end{abstract}

Laura Tapiovaara

laura.tapiovaara@hus.fi

1 Department of Otorhinolaryngology, Head and Neck Surgery, University of Helsinki and Helsinki University Hospital, PO Box 263, 00029 Helsinki, Finland

2 Department of Dentistry, University of California, Los Angeles, CA, USA need for tracheotomy seemed to lead to a longer hospital care and was associated with a more severe clinical course.

Keywords Airway management - Infection - Abscess · Tracheotomy $\cdot$ Head and neck

\section{Introduction}

Deep neck infections (DNI) continue to cause significant morbidity and mortality [1]. However, better overall health in the general population, developed health care systems, and therapeutic methods have improved the outcome [2]. DNI remains as a challenge for physicians because of the complexity of the anatomy in the head and neck area, vicinity of vital structures, and risk of spread of the infection into adjacent spaces such as the mediastinum, prevertebral and danger space, or intracranium.

The incidence of DNI is not well known. Major etiological factors in the adult population are odontogenic infections and pharyngotonsillitis, and salivary infections in the elderly [3]. Other possible etiological factors include trauma, iatrogenic factors such as prior surgery or procedure in the head and neck area, thyroglossal cyst or branchial cleft anomalies, intravenous drug use, and malignancies. Approximately in one-third of the cases the etiology remains unknown [4].

DNI may often compromise the airways. Thus, managing the airway appropriately is of utmost importance. It is crucial to consider that patient may experience trismus, and the anatomy of the neck may be distorted or show edema, which can further hinder conventional laryngoscopy and intubation. The exact location of the infection may also affect the choice of airway management [5]. The most often affected spaces regarding DNI include parapharyngeal, 
retropharyngeal, submental and sublingual, submandibular, buccal, carotid, and visceral spaces $[6,7]$.

The aim of the present study was to explore the causes and variation of management of DNI in a tertiary care center, with a specific focus on airway management in this patient group.

\section{Materials and methods}

This retrospective study was conducted in the University of Helsinki and Helsinki University Hospital, Helsinki, Finland, with a referral area of 1.6 million people. Patient data were retrieved from electronic data files of patients of 18 years or older between May 1, 2007 and December 31, 2016. We included all patients who underwent surgery at the Departments of Otorhinolaryngology, Head and Neck Surgery, Oral and Maxillofacial Surgery or Thoracic Surgery with an operative code ENA32 (which stands for: "Drainage of a deep neck infection") as a primary or secondary operative code. This operation code excludes the management of isolated peritonsillar abscesses and patients that are managed with aspiration only. Patients with a diagnosis of any malignancy within 5 years, and patients managed without the need for drainage of the abscess through the neck were excluded from further analysis. An institutional research permission was granted for the study protocol (HUS/356/2017 January 18, 2017).

Patient demographics, imaging, location of the infection, etiological factors, management of the airway, bacterial findings, length of stay in the intensive care unit (ICU) and hospital ward, postoperative complications, and follow-up were registered. Patient comorbidities were retrospectively evaluated from the hospital charts using comorbidity index ACE-27 [8]. Postoperative complications were evaluated retrospectively according to Clavien-Dindo classification [9].

We used descriptive statistics to summarize frequencies, proportions, medians, and ranges. Pearson's Chi-square test with asymptotic and exact $p$ values was used when best appropriate to calculate the statistical differences between categorical variables, and independent-samples $t$ test was used for continuous variables. A two-sided $p$ value of less than 0.05 was considered statistically significant. SPSS version 24 (SPSS, Inc. Chicago, IL, USA) served in all statistical analyses.

\section{Results}

In total, 231 patients underwent surgery because of DNI. From these, 29 (13\%) patients were excluded because of the following reasons: $15(7 \%)$ patients were operated through intraoral approach; $2(1 \%)$ had tuberculous cervical lymphadenitis requiring only excisional biopsy for diagnosis; $1(0.4 \%)$ had Bezold's abscess which was drained during mastoidectomy; $1(0.4 \%)$ had isolated cervical spinal abscess; $1(0.4 \%)$ patient received primary care outside our institution; and 9 (4\%) had a malignancy. This left 202 patients forming the study cohort. One patient was lost to follow-up in day 26 after admission because of transfer to a regional hospital. Most patients were operated on at the Department of Otorhinolaryngology, Head and Neck Surgery $(n=173 ; 86 \%)$. The Departments of Oral and Maxillofacial and Thoracic Surgery operated on 27 (13\%) and $2(1 \%)$ patients, respectively, in collaboration with head and neck surgeons. The management of patients with DNI includes close cooperation with all specialties involved in the treatment of these patients, especially when ICU care is required.

The baseline demographics and the method of primary airway management are presented in Table 1 .

\section{Preoperative imaging}

Computed tomography (CT) was the most common preoperative imaging modality $(n=165 ; 82 \%)$. Ultrasonography was utilized in 34 (17\%) patients and magnetic resonance imaging (MRI) in $12(6 \%)$ patients. No preoperative imaging was done in 13 (7\%) patients. More than one imaging modality was necessary for $22(11 \%)$ patients, and repeated preoperative imaging was considered necessary for $19(9 \%)$ patients.

\section{Etiology of deep neck infection}

Specific etiologies of DNI are presented in Table 2, and most common etiology was odontogenic infection $(n=72$; $36 \%)$. Thus, tooth extraction was performed for $59(29 \%)$ patients consecutively with the abscess drainage, and for 21 (10\%) during the postoperative days. Majority of extractions $(n=68 ; 85 \%)$ were in patients with known odontogenic etiology, and in $12(15 \%)$ patients because of poor oral hygiene. Four patients with known odontogenic etiology received dental care later at their own dental office. Bacteria from the abscess was identified in 105 (52\%) patients. Streptococcus anginosus was most common $(n=43 ; 21 \%)$, followed by Streptococcus pyogenes $(n=28 ; 14 \%)$. Bacteremia was identified in $26(13 \%)$ patients and $S$. pyogenes was most common $(n=9 ; 5 \%)$. The bacteria from blood cultures was different than bacteria from the abscess in 14 (13\%) patients. Table 3 shows the specific location of DNI and it occurred often as multilocular $(n=37 ; 18 \%)$. Patients were symptomatic for a median of 4 days (range 0-30) before admission. Surgery was executed within the first $24 \mathrm{~h}$ after admission for most patients $(n=135 ; 67 \%$; range $0-21)$. 
Table 1 Baseline demographics and the method of primary airway management

\begin{tabular}{lccc}
\hline & $\begin{array}{l}\text { All patients; } \\
n=202(\%)^{\mathrm{a}}\end{array}$ & $\begin{array}{l}\text { Intubation; } \\
n=165(\%)\end{array}$ & $\begin{array}{l}\text { Primary tracheotomy; } \\
n=35(\%)\end{array}$ \\
\hline Gender (\%) & & & $18(51)$ \\
Male & $127(63)$ & $108(65)$ & $17(49)$ \\
Female & $75(37)$ & $57(35)$ & 5.119 \\
Median age, years (range) & $47.0(18-86)$ & $46.0(18-86)$ & $7(4)$ \\
Diabetic (\%) & $19(9)$ & $11(7)$ & $18(51)$ \\
Comorbidity ACE-27 (\%) & & & $6(17)$ \\
0 & $107(53)$ & $89(54)$ & $3(9)$ \\
1 & $43(21)$ & $36(22)$ & $8(23)$ \\
2 & $27(13)$ & $23(14)$ & $0.003^{*}$ \\
3 & $25(12)$ & $17(10)$ & $0.012^{*}$ \\
Smoking (\%) & $37(18)$ & $32(19)$ & $8(11)$ \\
No & $46(23)$ & $38(23)$ & $10(3-56)$ \\
Yes & $7(2-80)$ & $6(2-80)$ & $4.5(1-27)$ \\
Median hospital stay (days; range) & $7(1-38)$ & $6(1-38)$ & 0.511 \\
Median length of ICU care (days; range) & & & $0.036^{*}$ \\
\hline
\end{tabular}

* Statistically significant

${ }^{a}$ Two patients who were treated in local anesthesia only

Table 2 Specific etiological factors for deep neck infections

\begin{tabular}{lll}
\hline Etiological factor & $\begin{array}{l}\text { Number }= \\
202\end{array}$ & Percentage (\%) \\
\hline Odontogenic & 72 & 36 \\
Tonsillar or pharyngeal & 18 & 9 \\
Iatrogenic factors $^{\mathrm{a}}$ & 15 & 7 \\
Lymphadenitis & 14 & 7 \\
Use of intravenous drugs & 10 & 5 \\
Sialadenitis & 10 & 5 \\
Infected cyst & 10 & 5 \\
Trauma & 4 & 2 \\
Epiglottitis & 3 & 1 \\
Hematogenic spread & 1 & 0.5 \\
Scalp eczema & 1 & 0.5 \\
Diabetic ketoacidosis & 1 & 0.5 \\
Spondylodiscitis & 1 & 0.5 \\
Agranulocytosis after clozapine use & 1 & 0.5 \\
Unknown & 41 & 20 \\
\hline
\end{tabular}

${ }^{a}$ Including dental procedures $(n=8)$; excision of the submandibular gland $(n=3)$; surgery for paraganglioma $(n=1)$; esophagogastroscopy $(n=1)$; unknown $(n=2)$

\section{Airway management with intubation}

DNI was managed in local anesthesia without the need for endotracheal intubation or tracheotomy in two patients. Intubation was the most common type of airway management in this patient group $(n=165 ; 82 \%)$, and extubation immediately after surgery often succeeded $(n=102 ; 50 \%)$.
Table 3 Location of deep neck infection determined radiologically and/or clinically

\begin{tabular}{llc}
\hline & $n=202$ & $\begin{array}{l}\text { Percent- } \\
\text { age }(\%)\end{array}$ \\
\hline Multilocular & 37 & 18 \\
Submental & 32 & 16 \\
Submandibular & 29 & 14 \\
Parapharyngeal & 25 & 12 \\
Necrotizing fasciitis in the & 16 & 8 \\
$\quad$ head and neck area & 15 & 7 \\
Retropharyngeal & 11 & 5 \\
Carotid space & 17 & 8 \\
Other & 20 & 10 \\
Unknown & & \\
\hline
\end{tabular}

${ }^{a}$ Infected branchial cleft cysts or thyroglossal duct cyst $(n=9 ; 4 \%)$, parotid $(n=3 ; 1 \%)$, visceral $(n=2 ; 1 \%)$, intramuscular $(n=2 ; 1 \%)$, nuchal $(n=1 ; 0.5 \%)$

Later tracheotomies were performed for 25 (15\%) patients in a median of 11 days (range 1-18) because of prolonged intubation and need for ICU care. However, there were 12 (19\%) patients who remained intubated for more than 7 days, and only six of them were tracheotomized in a median of 11 days (range 9-18) after the treatment onset. The remaining six patients remained intubated for a median of 11 days (range 8-26). Two patients were intubated for 26 days without the option for a tracheotomy for no obvious reason. The other patient had an odontogenic multilocular abscess. Another patient had multilocular DNI of unknown origin, 
Staphylococcus aureus sepsis, internal jugular vein thrombosis, and pulmonary empyema. The latter patient underwent surgery 5 times, 2 of which through thoracotomies. Infection that involved multiple locations and necrotizing fasciitis $(p<0.001)$ as well as extension to mediastinal space ( $p=0.004)$ prolonged the length of intubation in this cohort.

\section{Airway management with tracheotomy}

Primarily tracheotomized patients were older than primarily intubated $(p=0.003)$. Primary tracheotomy was performed for 35 (17\%) patients, and for $26(74 \%)$ of them in local anesthesia because of compromised airway. We utilize fiberoptic endoscopic examinations to evaluate whether patient has compromised airway. Head and neck surgeons performed 25 out of 26 tracheotomies, which were done in local anesthesia, and maxillofacial surgeons performed 1. Tracheotomy was maintained for a median of 8 days (range 1-32). Primary tracheotomy was offered for 13 (18\%) patients with an odontogenic etiology for DNI. DNI in the submental space increased the need for tracheotomy, and that was the method of primary airway management in 11 out of $32(34 \%)$ patients with DNI in this location $(p=0.005)$. Extension of the infection to mediastinal space increased the length of tracheotomy dependence $(p=0.007)$, and further the total length of hospital care $(p<0.001)$.

\section{Hospital stay}

Altogether $80(40 \%)$ patients were treated in the ICU for a median of 7 days (range 1-38). All intubated patients and who were not extubated immediately after surgery were treated at the ICU $(n=63 ; 31 \%)$. Intubated patients were treated at the ICU for a median of 6 days (range 1-38), and primarily tracheotomized patients for a median of 4.5 days (range 1-27), however, the difference was not statistically significant $(p=0.754)$. Median hospital care lasted for 7 days (range 2-80) and it was shorter for patients that were intubated (median 6 days; range 2-80) than for primarily tracheotomized (median 10 days, range 3-56; $p=0.036$ ). However, patients that were intubated and not extubated immediately after surgery $(n=63 ; 31 \%)$, were treated at the hospital for a median of 13 days (range 3-80), but the difference was insignificant compared to patients that got tracheotomy primarily $(p=0.391)$.

\section{Complications related to deep neck infection}

In $25(12 \%)$ patients the infection extended to the mediastinal space, and most of these occurred in patients with odontogenic infection ( $n=12 ; 48 \%)$, and with necrotizing fasciitis $(n=8 ; 32 \%)$ although these associations failed to show statistical significance. Further, 9 (56\%) patients with necrotizing fasciitis had an etiology of an odontogenic infection, and the mediastinum was involved in 6 (67\%) of them. We found no association between tracheotomy and the development of mediastinitis in any patient $(p=0.815)$. Mediastinitis developed in $5(14 \%)$ primarily tracheotomized patients and in $20(12 \%)$ primarily intubated patients. Re-canalization of the abscess was required for $44(22 \%)$ patients, and for $81 \%$ of patients with necrotizing fasciitis. A postoperative complication required surgical intervention in 5 (2\%) patients. The complications were of grade IIIb (postoperative bleeding; $n=3)$ and of grade IV $(n=2)$. The two more severe complications included a sudden subarachnoid hemorrhage after tooth extraction that required craniotomy twice, and carotid artery bleeding a few hours after initial surgery, which was managed with vena saphena magna bypass. Both of these patients were primarily treated by head and neck surgeons but cooperation with neurosurgeon and vascular surgeon was necessary in the management of these complications. Recurrent laryngeal nerve paralysis occurred in $3(1 \%)$ patients and was managed conservatively. Tracheotomy did not increase the complication rate. In-hospital mortality rate was $1 \%(n=2)$. A 86 -year-old female with odontogenic retropharyngeal abscess died 25 days postoperatively after developing hypernatremia and cardiopulmonal dyspnea. A 58-year-old male died 3 days after hospitalization of necrotizing fasciitis and $S$. pyogenes sepsis, which caused disseminated intravascular coagulation and severe renal dysfunction.

\section{Discussion}

We present a retrospective study of 202 adult patients with DNI and who were managed surgically in a tertiary care hospital during a 10-year period. We specifically aimed at evaluating airway management in this challenging patient group. Compromised airway was encountered in 13\% of patients and odontogenic infections predominated. DNIs result in high costs of care. We had $40 \%$ of patients in need for ICU care, and the median hospital stay was 7 days with a maximum of over 2 months.

A study by Barber et al. [10] explored etiological factors associated with a more severe clinical course of DNI. These factors included lower level of education, residing further than an hour distance from tertiary care center, remaining tonsils, and Streptococcal infections. We showed that odontogenic infections were a common etiology in necrotizing fasciitis and in mediastinal extension of the infection, and thus presented with a more severe course of the disease. Similar notifications are reported by Kinzer et al. [11]. Diabetes mellitus (DM) seems to increase the risk for lifethreatening complications [12], but the number of patients 
with DM in the present study was too low to draw any conclusions.

In DNI, tracheotomy has been stated as the gold standard in the management of compromised airway [7]. Modern airway management, including utilization of video-laryngoscope and fiberoptic bronchoscope can, however, result in successful intubation [5]. We used primary tracheotomy in $35(17 \%)$ patients, and $25(12 \%)$ of intubated patients were tracheotomized later. Some previous studies present a rather low tendency to perform tracheotomy in this patient group. A study by Motahari et al. [13] including 815 cases reported $1 \%$ of patients to receive a tracheotomy, and $5 \%$ of patients received a tracheotomy in a study by Kataria et al. [14] including 76 cases. Both studies included peritonsillar abscesses, which probably explains the low incidence of tracheotomy. Our study excluded peritonsillar abscesses since they are managed generally intraorally.

Our study shows that submental space was a common location of DNI $(n=32 ; 16 \%)$ and those patients got a tracheotomy more often than patients with DNI in other locations. Ludwig's angina, a bilateral infection of the submandibular and sublingual compartments of the neck and floor of the mouth, often presents with obstructed airway [15]. A study by Botha et al. [16] reports high rates of tracheotomy (66\%) in patients with Ludwig's angina, which exceeds our rates (34\%). However, we cannot confirm that all our patients with submental involvement of DNI had Ludwig's angina.

Most of the patients in the present study were operated within the first day after admission. A recent study showed that a delay in surgical drainage of DNI was associated with higher morbidity and mortality [17]. On the contrary, a study by Boscolo-Rizzo et al. [18] reported that $62 \%$ of patients responded to antimicrobial treatment only. In that study, however, patients with conservative management approach were in stable condition and included cellulitis, abscesses less than $3 \mathrm{~cm}$ in diameter and limited to only 1 space, and no danger space involvement. Further, conservative therapy was continued only if both clinical signs and symptoms, and CT showed improvement.

It seems that the need for tracheotomy indicates a more severe course of the disease. In the present study patients with a tracheotomy required longer hospital care, which we feel is the result of the disease and not because of the chosen method of airway management. A study by Barber et al. [10] also reported that airway compromise prolongs hospitalization. However, primarily tracheotomized patients showed a trend for shorter ICU care compared to intubated and not immediately extubated patients, although this difference was statistically insignificant. A study by Potter et al. [19] reported similar results, and they also showed tracheotomy to reduce the total cost of the care. We had 12 patients who were intubated for over 7 days and two patients were intubated for 26 days without offering a tracheotomy, and this most likely prolonged their ICU care. There is no consensus on the optimal timing of tracheotomy after a prolonged intubation [20].

A study by Chen et al. [21] underlines the difficulty to perform tracheotomy in DNI and the risk of complications related to the procedure. We present a rather high number of compromised airway since $13 \%$ of patients were tracheotomized in local anesthesia. However, we found no complications related to tracheotomy. Tracheotomy can offer several benefits in ICU care compared to intubation. These include for example avoiding laryngeal injury caused by prolonged intubation, facilitating nursing care, improving patient comfort by reducing the need for sedation, and reducing the need for mechanical ventilation [22]. An early tracheotomy within 7 days after intubation seems to reduce the duration of artificial ventilation and length of stay at the ICU [20]. A study by Mahafza et al. [23] compared early and late tracheotomy and found tracheotomy in less than 3 weeks after intubation to hinder complications and lower mortality at the ICU.

A study by Celakovsky et al. [24] reported extension of DNI to mediastinal space to increase morbidity and mortality. They found additional risk factors for mediastinal involvement that included pulmonary and cardiovascular comorbidities, and origin of the infection in the tonsil. Necrotizing fasciitis is a severe disease with often sudden onset and rapid progression. We found that odontogenic etiology and necrotizing fasciitis had a higher tendency, although not statistically significant, for involvement of the mediastinal space. Tracheotomy did not associate with the development of mediastinitis. In cardiac surgery, tracheotomy seems to increase the risk for mediastinitis [25, 26]. Recommendations to perform tracheotomy remain indefinite in DNI, but we found it safe in this study cohort. Not only did mediastinal involvement prolong the need for hospitalization in the present study, but it also increased complications as three patients developed recurrent laryngeal nerve paralysis and one patient succumbed.

We acknowledge the limitations in the present study, which are mostly due to the retrospective study design. The charts did not allow us to confirm the results of the microbiology in all cases. Similarly, the individual consideration on the choice of the airway management was not always possible to evaluate. The three centers included in the present study solely treat DNI patients in the whole referral area providing a vast coverage on the management of DNI. Further, same centers that managed the DNI managed also the complications and follow-up. 


\section{Conclusions}

The possible life-threatening sequelae of DNI need special attention. Since mediastinal extension occurs quite frequently in this patient group and associates with higher morbidity and mortality, it is crucial to diagnose and manage it appropriately. Odontogenic infection remains as a risk factor for the spread of infection. In addition, we found the need for tracheotomy to associate with a more severe clinical course of the disease. Whether to perform endotracheal intubation or tracheotomy requires individual assessment and cooperation with the anesthesiologist, and we found tracheotomy to be safe. The demand for ICU care consumes resources and increases costs of care. In DNI, tracheotomy may decrease the need for ICU care and decrease complications related to longer intubation periods.

\section{Compliance with ethical standards}

Conflict of interest Author LT declares that she has no conflict of interest. Author LB declares that he has no conflict of interest. Author KA declares that she has no conflict of interest.

Ethical approval This article does not contain any studies with human or animal participants performed by any of the authors. This research involved only patient charts. For this type of study formal consent is not required.

Funding This study was supported by grants from the Sigrid Jusélius Foundation and the Finnish Otorhinolaryngology Research Foundation.

\section{References}

1. Wang LF, Kuo WR, Tsai SM, Huang KJ (2003) Characterizations of life-threatening deep cervical space infections: a review of one hundred ninety-six cases. Am J Otolaryngol 24:111-117

2. Boscolo-Rizzo P, Marchiori C, Montolli F, Vaglia A, Da Mosto MC (2006) Deep neck infections: a constant challenge. ORL J Otorhinolaryngol Relat Spec 68:259-265

3. Chi TH, Tsao YH, Yuan CH (2014) Influences of patient age on deep neck infection: clinical etiology and treatment outcome. Otolaryngol Head Neck Surg 151:586-590

4. Parhiscar A, Har-El G (2001) Deep neck abscess: a retrospective review of 210 cases. Ann Otol Rhinol Laryngol 110:1051-1054

5. Cho SY, Woo JH, Kim YJ et al (2016) Airway management in patients with deep neck infections: a retrospective analysis. Medicine (Baltimore) 95:e4125

6. Levitt GW (1970) Cervical fascia and deep neck infections. Laryngoscope 80:409-435

7. Karkos PD, Leong SC, Beer H, Apostolidou MT, Panarese A (2007) Challenging airways in deep neck space infections. Am J Otolaryngol 28:415-418

8. Piccirillo JF, Johnston A, Claybour P, Littenberg B, Creech C, Anderson S, Grove L, Costas I (2003) Comorbidity coding book. Washington University School of Medicine, St. Louis
9. Dindo D, Demartines N, Clavien PA (2004) Classification of surgical complications: a new proposal with evaluation in a cohort of 6336 patients and results of a survey. Ann Surg 240:205-213

10. Barber BR, Dziegielewski PT, Biron VL, Ma A, Seikaly H (2014) Factors associated with severe deep neck space infections: targeting multiple fronts. J Otolaryngol Head Neck Surg 43:35

11. Kinzer S, Pfeiffer J, Becker S, Ridder GJ (2009) Severe deep neck space infections and mediastinitis of odontogenic origin: clinical relevance and implications for diagnosis and treatment. Acta Otolaryngol 129:62-70

12. Hidaka H, Yamaguchi T, Hasegawa J et al (2015) Clinical and bacteriological influence of diabetes mellitus on deep neck infection: systematic review and meta-analysis. Head Neck 37:1536-1546

13. Motahari SJ, Poormoosa R, Nikkhah M, Bahari M, Shirazy SM, Khavarinejad F (2015) Treatment and prognosis of deep neck infections. Indian J Otolaryngol Head Neck Surg 67:134-137

14. Kataria G, Saxena A, Bhagat S, Singh B, Kaur M, Kaur G (2015) Deep neck space infections: a study of 76 cases. Iran J Otorhinolaryngol 27:293-299

15. Candamourty R, Venkatachalam S, Babu MR, Kumar GS (2012) Ludwig's Angina-an emergency: a case report with literature review. J Nat Sci Biol Med 3:206-208

16. Botha A, Jacobs F, Postma C (2015) Retrospective analysis of etiology and comorbid diseases associated with Ludwig's Angina. Ann Maxillofac Surg 5:168-173

17. Cramer JD, Purkey MR, Smith SS, Schroeder JW Jr (2016) The impact of delayed surgical drainage of deep neck abscesses in adult and pediatric populations. Laryngoscope 126:1753-1760

18. Boscolo-Rizzo P, Stellin M, Muzzi E et al (2012) Deep neck infections: a study of 365 cases highlighting recommendations for management and treatment. Eur Arch Otorhinolaryngol 269:1241-1249

19. Potter JK, Herford AS, Ellis E (2002) Tracheotomy versus endotracheal intubation for airway management in deep neck space infections. J Oral Maxillofac Surg 60:349-354 (discussion 354-355)

20. Griffiths J, Barber VS, Morgan L, Young JD (2005) Systematic review and meta-analysis of studies of the timing of tracheostomy in adult patients undergoing artificial ventilation. BMJ 330:1243

21. Chen SJ, Ji N, Chen YX et al (2015) Management and maintenance of the airway in cervical necrotising fasciitis: a retrospective analysis of 15 cases. Br J Oral Maxillofac Surg 53:642-646

22. Blot F, Similowski T, Trouillet JL et al (2008) Early tracheotomy versus prolonged endotracheal intubation in unselected severely ill ICU patients. Intensive Care Med 34:1779-1787

23. Mahafza T, Batarseh S, Bsoul N, Massad E, Qudaisat I, Al-Layla AE (2012) Early vs. late tracheostomy for the ICU patients: experience in a referral hospital. Saudi J Anaesth 6:152-154

24. Celakovsky P, Kalfert D, Tucek L et al (2014) Deep neck infections: risk factors for mediastinal extension. Eur Arch Otorhinolaryngol 271:1679-1683

25. Curtis JJ, Clark NC, McKenney CA et al (2001) Tracheostomy: a risk factor for mediastinitis after cardiac operation. Ann Thorac Surg 72:731-734

26. Pilarczyk K, Marggraf G, Dudasova M et al (2015) Tracheostomy after cardiac surgery with median sternotomy and risk of deep sternal wound infections: is it a matter of timing? J Cardiothorac Vasc Anesth 29:1573-1581 